\title{
Improved Detection Scheme for Chipless RFIDs Using Prolate Spheroidal Wave Function-based Noise Filtering
}

\author{
Wouter Dullaert, Lars Reichardt, Student Member IEEE Hendrik Rogier, Senior Member IEEE
}

\begin{abstract}
A novel, highly sensitive scheme to detect the resonance peaks in the spectrum of chipless RFID signal is presented. The detection is based on finding the zeros in the derivative of the group delay of the received signal. In order to be able to accurately detect these zeros in the presence of noise the received signal is filtered using a Prolate Spheroidal Wave Function based model. This allows great increases in the distance at which chipless RFIDs can be accurately read. The detection method can be used standalone or in addition to traditional amplitude based detection schemes.
\end{abstract}

Index Terms-UWB, PSWF, RFID

\section{INTRODUCTION}

Radio Frequency Identification (RFID) technology can be roughly divided into two categories: RFIDs with an Integrated Circuit (IC) on the tag, and so-called chipless RFIDs. In recent years, RFID tags with ICs have seen major adoption in various industries, because of their robust performance, decent reading range and relatively low price. However battery life (if the IC has a power source) and the cost of the IC prevent the RFID from becoming the new barcode.

In order to overcome these issues recent research activity has shifted towards fully passive, chipless RFIDs. However, due to the lack of an internal power supply the operation range is drastically reduced. Furthermore the chipless RFID has no means to adapt itself to a more difficult propagation channel. These difficulties have made Ultra Wideband (UWB) techniques a popular choice for these kinds of RFID systems:

1) The low spectral energy density allows the system to coexist with other wireless systems.

2) The aggregated energy over the bandwidth results in an acceptable overall signal level.

3) The large bandwidth of pulse based systems makes the system almost immune to multipath fading.

Because the RFID tag itself has no power source, it must use the power of the incident field to transmit its data. Various ways of encoding data into the tag have been presented. For instance, [1], [2] encode the data into the phase of the backscattered signal. The tag emits two signals using frequency or polarisation diversity: a signal providing a reference phase and a signal with a phase difference that depends on the tag. The amount of bits that can be stored by this sort of tag depends on the resolution at which the phase difference can be reliably resolved at the tag reader. In [3] a pulse position modulation technique is used. Based on which pulses from the emitted signal are delayed, a bitstring can be decoded.
Others, such as [4], [5], operate on the amplitude of the frequency spectrum by notching out certain frequencies in the interrogation signal.

In this letter a new method is proposed to detect the data transmitted by chipless RFIDs that rely on this last frequencydomain method, by detecting the zeros in the derivative of the group delay. Using a model based on Prolate Spheroidal Wave Functions (PSWFs) the noise in the group delay is reduced, making this a viable approach. It allows to read the tags up to a distance of $10 \mathrm{~cm}$, which was previously impossible.

In Section II the RFID tags under consideration are briefly described. The new detection method and PSWF-based model are presented in Section III. Results of the new method are compared to the traditional method in Section IV. Finally, the conclusions are presented in Section V.

\section{ChIPLESS RFID TAG}

This letter relies on measured data from RFID tags presented in [4]. These tags are fully passive without ICs or internal power supply. A schematic of the entire system is shown in Fig. 1. The tag reader transmits an interrogation signal onto which the tag encodes its data using a series of resonators to notch out certain frequencies in the spectrum of the interrogation signal. The coded signal is transmitted along a polarisation orthogonal to the polarisation of the receive antenna in order to minimize interference. The signal is then processed by the tag reader, where notched out frequencies represent a logic zero, the other frequencies a logic one. Measurements of the passive tag can be easily performed by doing a frequency sweep with a network analyser at various distances, [4].

The RFID tag consists of orthogonally polarised UWB transmit and receive antennas connected to a microstrip line. Resonators are placed besides this microstrip line, suppressing certain frequencies in the signal that propagates along the microstrip line, as shown schematically in Fig. 2. The resonance frequency of the resonators can be changed by manipulating the overall length $l$ of the spiral (the dotted part of the spiral on Fig. 2). The other dimensions shown in Fig. 2 are constant for all resonators. The amount of information encoded into such a tag is determined by the bandwidth of the signal and the quality of the resonators. The tag itself operates between $3.9 \mathrm{GHz}$ and $4.4 \mathrm{GHz}$ over a bandwidth of $500 \mathrm{MHz}$ and is therefore compliant with the UWB specification.In this band, the UWB antenna has a flat response, but the spiral resonators 


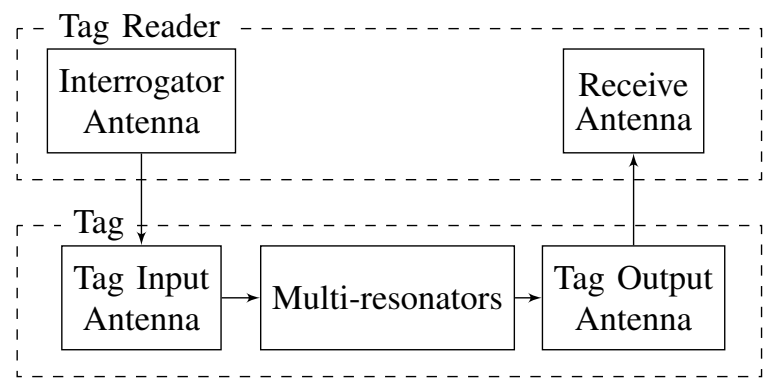

Fig. 1. RFID System Schematic

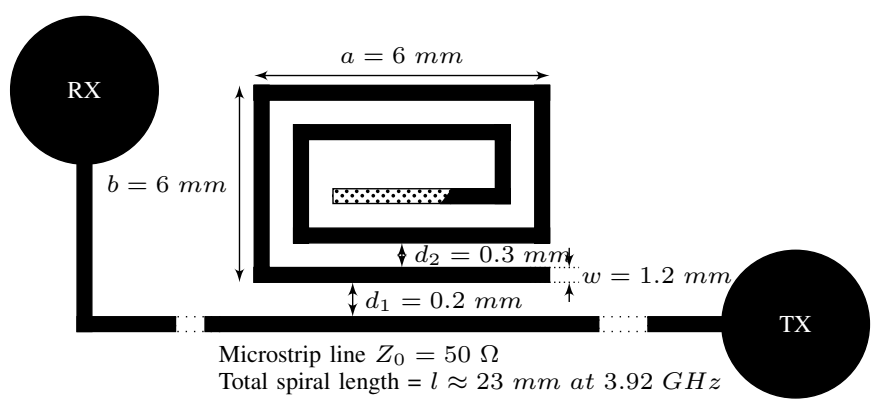

Fig. 2. Schematic of a spiral resonator, from [4]

make the tag resonate at $3.92,3.96,4.06,4.11,4.2,4.28$ and $4.36 \mathrm{GHz}$, storing 7 bits. The response of the $0000000 \mathrm{tag}$, resonating at all specified frequencies, measured at $5 \mathrm{~cm}$, is shown in Fig. 3.

The tag information is decoded by comparing the normalised spectrum of the received pulse to a reference signal, in this case the normalised spectrum of a 0000000 tag. If the difference between the received and reference signal exceeds a threshold of $5 \mathrm{~dB}$ at a resonance frequency, the bit is considered to be a logic one. This method of detection is shown in Fig. 4 for a 0110001 tag at $5 \mathrm{~cm}$ distance. The black lines correspond to logic zeros, the dashed red lines to logic ones. The signal has already been processed by a time gating algorithm, [4] to improve detection and overcome coupling between the receiver antennas. Bit 2 and 7 are correctly detected as logic ones, whereas bit 3 is not. Adjusting the threshold will not solve the problem because bit 4 will then be wrongly decoded as a logic one.

It is obvious that this decoding technique has poor performance. It only uses the amplitude of the signal, making it very sensitive to interference with other signals near the resonance frequencies of the tag. Also path loss caused by the increased distance between the tag and reader makes it hard to determine a good value for the detection threshold. The performance can be improved by also considering the phase of the signal. Such a detection scheme is presented in the following section.

\section{Phase-BASED Detection}

\section{A. Detection Scheme}

In addition to relying on the amplitude characteristic of the received signal, a technique that uses the phase of the signal to detect the resonances in the tag is proposed. The spiral

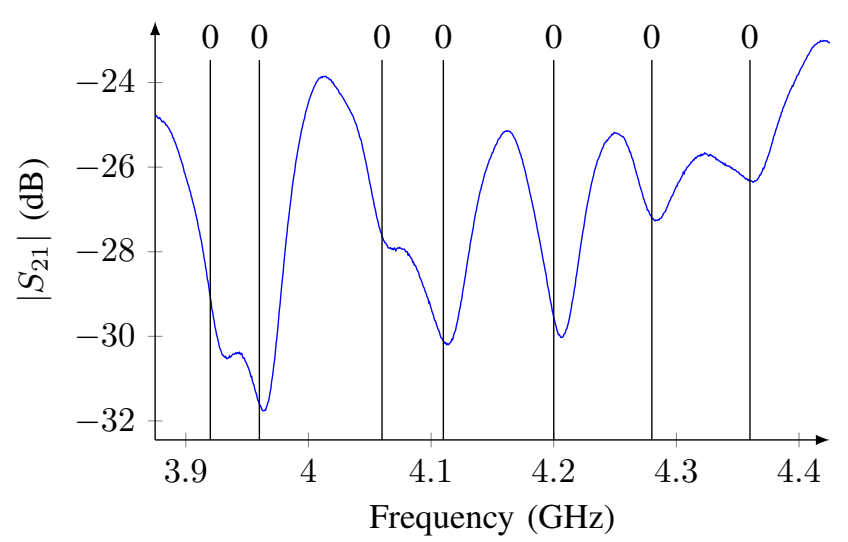

Fig. 3. Amplitude of the response of a 0000000 tag

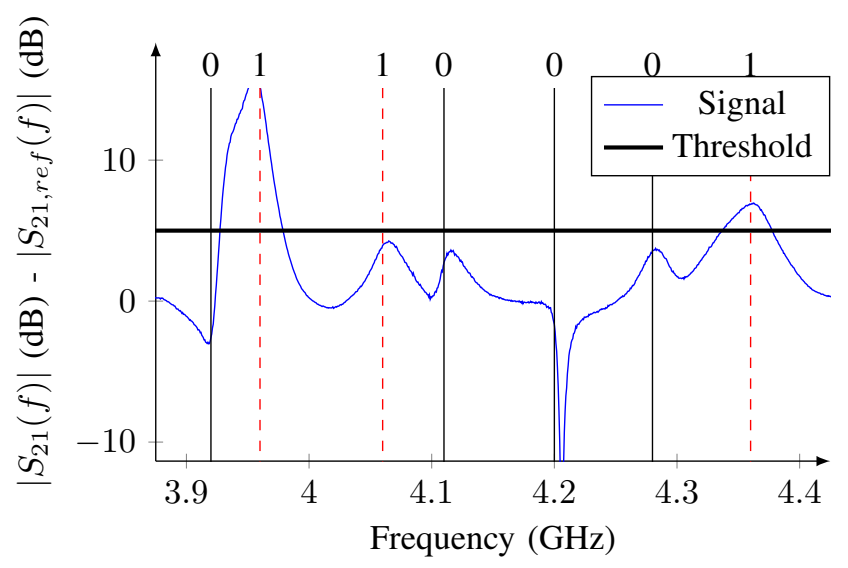

Fig. 4. Classic tag decoding result for a 0110001 tag

resonators that encode the bits into the tag spectrum are notch filters that cause a spike in the phase response. The largest rate of change is found at the resonance frequency of the filter: the group delay of the signal exhibits a maximum at the resonance frequency. This means that by finding the zeros on a falling edge of the second-order derivative of the phase of the received signal, the resonance frequencies can be detected. It is assumed that the detector knows the location of the potential resonances in the tags.

Due to various factors, such as production variability, the resonance frequency can deviate a bit from the specified frequency. This can be seen in Fig. 3: the minima of the amplitude do not perfectly correspond to the predefined resonance frequency (the vertical lines). In order to compensate for this, a small band around the resonance frequency $f_{\text {res }}$ : $f_{\text {res }}-\epsilon<f<f_{\text {res }}+\epsilon$ is investigated. The choice of $\epsilon$ is critical for the accuracy of the system and depends on the minimum spacing between the resonance frequencies and total bandwidth available. As a rule of thumb $\epsilon=0.003 \times f_{c}$ is used, with $f_{c}$ the center frequency of the used frequency band.

Unfortunately the noise present in any measured signal will increase drastically in its derivatives. This can be seen in Fig. 5, which shows the second-order derivative of the phase response of a 0000000 tag. However, we propose to combine this detection method with a filtering technique that 


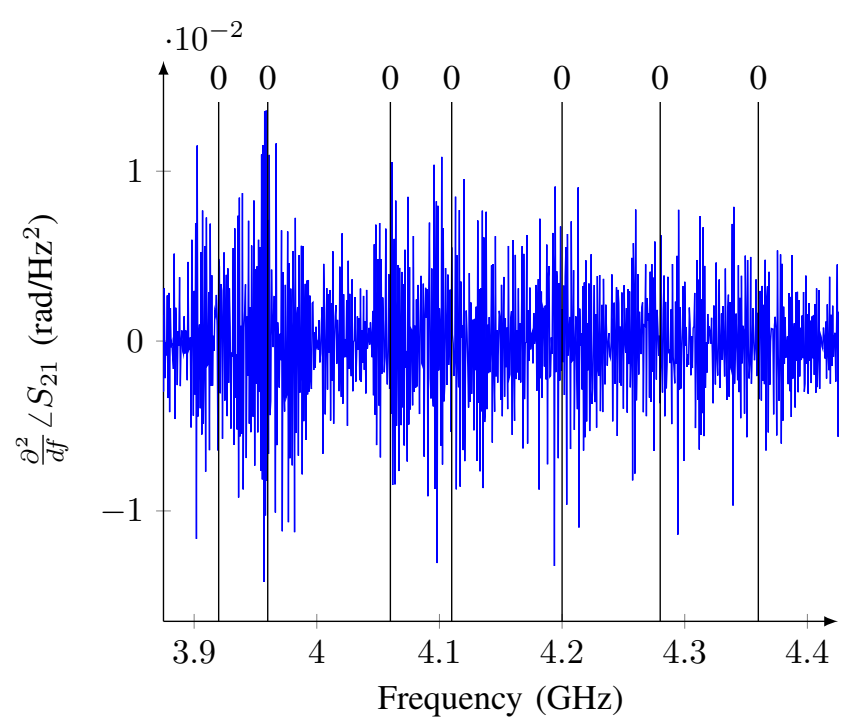

Fig. 5. Second derivative of the phase-response of the 0000000 tag

results in signals that are smooth enough to allow accurate detection of the zeros in the second-order derivative of their phase characteristic.

\section{B. PSWF-based Filter}

In order to reduce the noise contribution of the measured phase response, the received phase signal is modeled using a series of Prolate Spheroidal Wave Functions (PSWFs), [6], [7]. These functions arise from the following maximisation problem, over all bandlimited functions:

$$
\alpha^{2}(T)=\frac{\int_{-T / 2}^{T / 2} f^{2}(t) d t}{\int_{-\infty}^{\infty} f^{2}(t) d t}
$$

This means that the PSWFs are the bandlimited functions with the most energy concentration in a given time interval $[-T / 2, T / 2]$. This makes these PSWFs extremely suited to model bandlimited signals. The received phase $\Phi(f)$, being a function of frequency, is expanded into a truncated series of PSWFs $\psi(f)$, as in [8] but for the continuous case:

$$
\Phi(f)=\sum_{k=0}^{K} A_{k} \psi(f)
$$

As PSWFs are specifically tailored to describe bandlimited signals, only a very limited amount of coefficients is needed to accurately describe the actual signal. The measurement noise, however, is typically not bandlimited: it requires a large amount of coefficients. By truncating the series in an appropriate manner the noise is filtered out and a smooth signal remains. Once the coefficients for the model are obtained, the first and second-order derivative of the signal are calculated:

$$
\frac{\partial^{2}}{\partial f} \Phi(f)=\sum_{k=0}^{K} A_{k} \frac{\partial^{2}}{\partial f} \psi(f)
$$

In Fig. 6 the coefficients of the phase response of the 0000000 tag are shown. It is seen that the amplitude of the

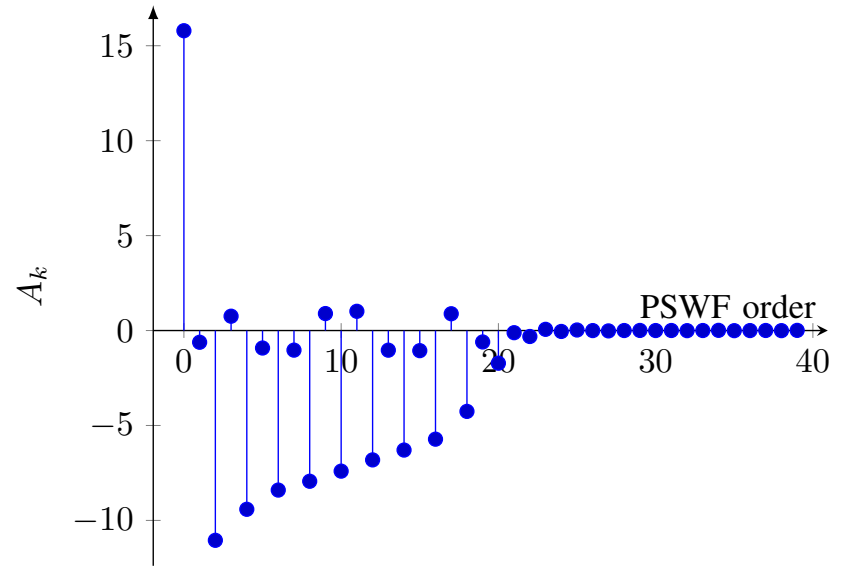

Fig. 6. Model coefficients of the phase-response of the 0000000 tag

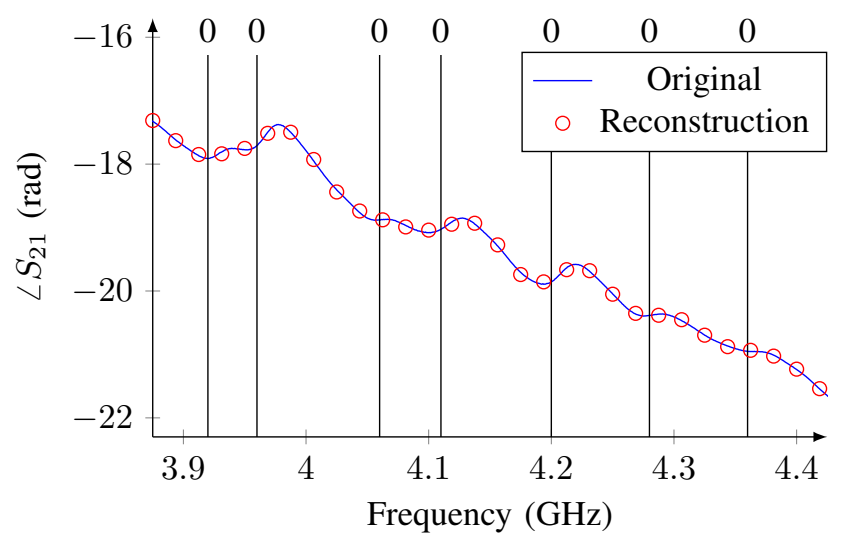

Fig. 7. Reconstruction of the phase response of the 0000000 tag

coefficients is almost zero above order 25: the model provides a very compact representation of the signal. In Fig. 7 both the original and reconstructed phase response are shown. A very good agreement is obtained. However the reconstructed signal is much smoother than the original. This is verified on Fig. 8 , showing the second-order derivative of the reconstructed signal. The excessive noise from Fig. 5 is removed and the zeros on falling edges can now be detected. Furthermore the receiver does not need a reference signal. Only the location of the potential resonances is needed to obtain accurate detection.

\section{RESUlts}

This new detection scheme is tested on 2 different tags: a 0000000 tag and a 0110001 tag. In all relevant figures, the following convention is used: the full black vertical lines mark frequencies at which the tag resonates. The dashed red lines indicate an absence of a resonance. The dotted lines indicate the guard inteval with a $2 \epsilon$ width around a resonance frequency. Using the rule of thumb, $\epsilon$ becomes: $\epsilon=0.003 \times 4.15 \mathrm{GHz} \approx 12.45 \mathrm{MHz}$.

\section{A. 0000000 Tag}

The filtered second-order derivative of the phase for the $0000000 \mathrm{tag}$, measured at $5 \mathrm{~cm}$ distance, has already been shown in Fig. 8. All resonances, except the first one, have 


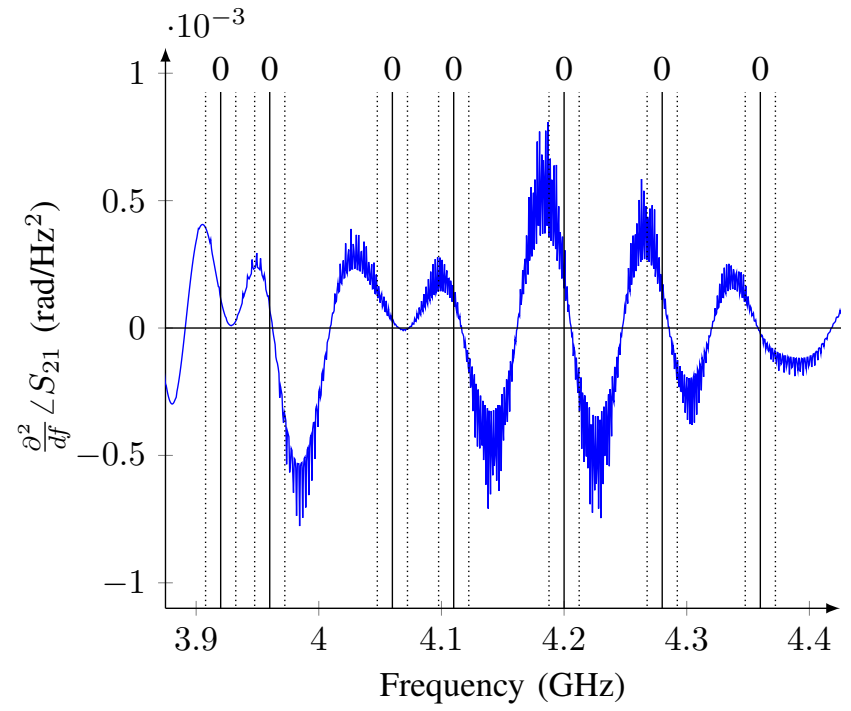

Fig. 8. Filtered second derivative of the phase-response of the 0000000 tag

a zero on a falling edge within the guard interval. This tag highlights the most important condition for this detection scheme to work: the spacing between the resonances must be large enough so that they can be individually recognised. When the resonances are too close together, such as the first and second or third and fourth, they start to appear as one resonance and cannot be accurately distinguished: neither by this method, nor by the the traditional amplitude based methods. This is an issue that needs to be considered during the tag design.

\section{B. $0110001 \mathrm{Tag}$}

The filtered second-order derivative of the phase response for the $0110001 \mathrm{tag}$ is shown in Figs. 9 and 10, measured at $5 \mathrm{~cm}$ and $10 \mathrm{~cm}$ respectively. As this tag does not contain any resonances too close to each other, all falling edges of the second-order derivate of the phase response are clearly visible and located at the right frequencies. At a measurement distance above $10 \mathrm{~cm}$ decoding errors start to occur. This result is a great improvement over the traditional method that failed to decode this tag, even at $5 \mathrm{~cm}$.

\section{CONCLUSION}

A new method for detecting the resonances in spectral encoded chipless RFIDs has been presented. The new method relies on the second-order derivative of the phase of the signal instead of its amplitude and does not require a reference signal. In order to be able to calculate the second-order derivative a PSWF-based filter is applied to the phase of the signal. The new method allows accurate detection of the resonances in the received signal, for distances of up to $10 \mathrm{~cm}$, whereas the traditional method cannot detect the presence or absence of all resonances, even when measured at $5 \mathrm{~cm}$. In future work the PSWF-based filtering technique will be compared to similar approaches such as wavelet based denoising.

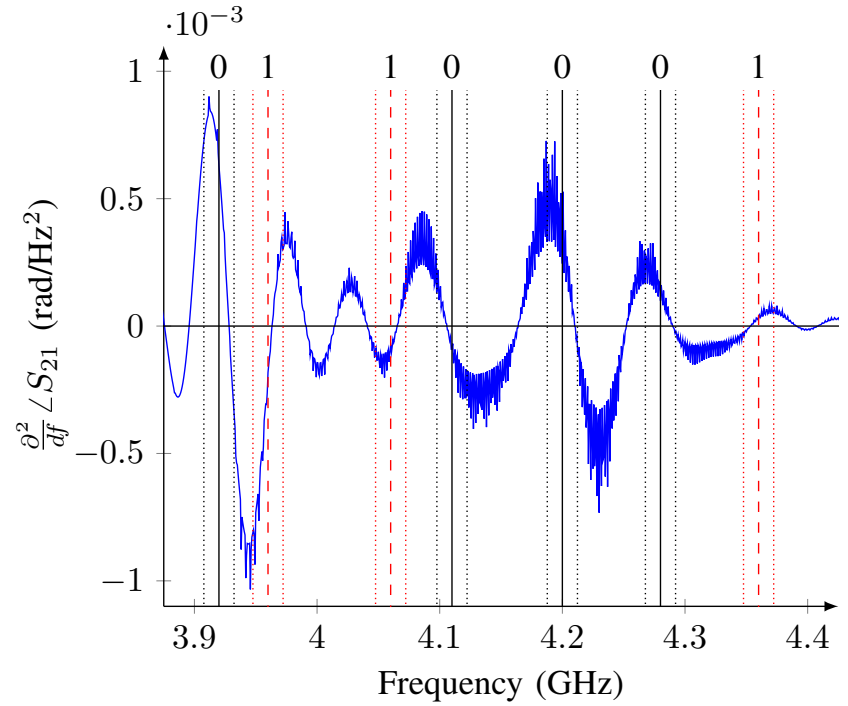

Fig. 9. Second-order derivative of the 0110001 tag phase-response at $5 \mathrm{~cm}$

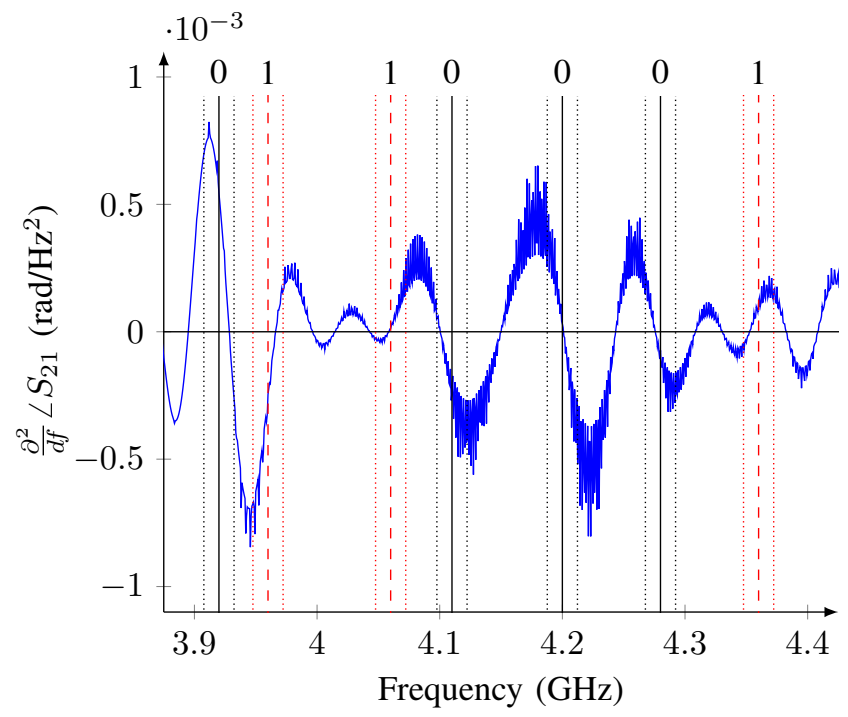

Fig. 10. Second-order derivative of the 0110001 tag phase-response at 10 $\mathrm{cm}$

\section{REFERENCES}

[1] S. Mukherjee, "Chipless RFID using stacked multilayer patches," 2009 Applied Electromagnetics Conference (AEMC), pp. 1-4, Dec. 2009.

[2] I. Balbin and N. C. Karmakar, "Phase-Encoded Chipless RFID Transponder for Large-Scale Low-Cost Applications," IEEE Microwave and Wireless Components Letters, vol. 19, no. 8, pp. 509-511, Aug. 2009.

[3] S. Gupta, B. Nikfal, and C. Caloz, "RFID System based on Pulse-Position Modulation using Group Delay Engineered Microwave C-Sections," in Asia Pacific Microwave Conference 2010, Yokohoma, Japan, 2010.

[4] L. Reichardt, G. Adamiuk, G. Jereczek, and T. Zwick, "Car-toinfrastructure communication using chip-less, passive RFID tags," in 2010 IEEE Antennas and Propagation Society International Symposium. Toronto, Ontario, Canada: IEEE, Jul. 2010, pp. 1-4.

[5] S. Preradovic and N. Karmakar, "Design of fully printable planar chipless RFID transponder with 35-bit data capacity," in European Microwave Conference, 2009., no. October, 2009, pp. 13-16.

[6] D. Slepian and H.O. Pollak, "Prolate Spheroidal Wave Functions, Fourier Analysis, and Uncertainty - I," Bell Systems Technology Journal, vol. 40, no. 1, pp. 43-64, Jan. 1961.

[7] M. Abramowitz and I. Stegun, Handbook of Mathematical Functions. Dover Publications, 1972, pp. 751-759.

[8] W. Dullaert and H. Rogier, "Novel Compact Model for the Radiation Pattern of UWB Antennas Using Vector Spherical and Slepian Decomposition," IEEE Transactions on Antennas and Propagation, vol. 58, no. 2, pp. 287-299, 2010. 